\title{
The Significance to Determine Factors Related to Postoperative Cerebral Infarction in Patients with Moyamoya Disease
}

\author{
Jin Yu Jibo Zhang Jincao Chen \\ Department of Neurosurgery, Zhongnan Hospital of Wuhan University, Wuhan, China
}

Dear Editor,

We read with great interest the article by Qian et al. [1] regarding factors related to cerebral infarction after direct bypass surgery in adults with moyamoya disease (MMD). The authors found that the history of preoperative cerebral infarction and the Suzuki stages of the nonoperative side were associated with an increased probability of cerebral infarction after surgery. We really appreciate the interesting observation for their conclusion. However, after reading this article, we would like to highlight 2 important questions that it raises.

First, the relationship between the preand postoperative infarction regions should be clarified during the analyses. Previous studies have shown that the age, preoperative cerebral infarction, Suzuki stages, and so on were risk factors of postoperational cerebral infarction in MMD [2]. However, the mechanisms and patterns are not clear yet. In addition to the management of anesthesia discussed by the authors in the present study, there are 2 possibilities in our opinion: (1) The sudden extracranial blood flow which is induced by the bypass surgery interferes with the intracranial hemodynamic balance and the regulation of intracranial blood flow distribution, which led to cerebral infarction. For example, watershed shift phenomenon [3]. (2) Because of the progressive occlusion of the main cerebellar arteries and decreased flow from the ineffective moyamoya vessels, the bypass operation may not effectively compensate the perfusion of the ischemic area. In our latest observations [4], we observed different hemodynamic sources of the recipient parasylvian cortical arteries (PSCA) among the frontal, temporal, and parietal PSCAs in MMD hemispheres. Different recipient PSCAs in the direct bypass surgery have different effect for different brain areas. All these conditions need to be determined by the area of the postoperational infarction.

Second, the authors found a significant risk factor related to the cerebral infarction after surgery - the Suzuki stages of the nonoperative side. Indeed, as reported by Bonasia et al. [5], in order to compensate the perfusion of ischemic areas, there exists a wide vascular anastomosis in the brain of MMD patients. Thus, operation on the more systematically unstable side may more induce a loss of moyamoya vessels, which may eventually affect cerebral blood flow in the opposite hemisphere.
This is of great significance for the choice of treatment strategy for bilateral MMD patients.

\section{Acknowledgment}

The authors thank all participants in the study.

\section{Disclosure Statement}

The authors have no conflicts of interest to declare.

\section{Funding Sources}

This research did not receive any specific grant from funding agencies in the public, commercial, or not-for-profit sectors.

\section{Author Contributions}

J.Y. and J.Z. performed investigation, review, and writing. J.C. responded for supervision.

\section{KARGER}

(c) 2020 S. Karger AG, Basel

karger@karger.com

www.karger.com/ced
Jincao Chen

Department of Neurosurgery

Zhongnan Hospital of Wuhan University

Donghu Road 169, Wuhan 430071 (China)

E-Mail yokinns@163.com 


\section{References}

1 Qian Y, Huang B, Hu Z, Wang J, Zhao P, Li $X$. Analysis of Factors Related to Cerebral Infarction after Direct Bypass Surgery in Adults with Moyamoya Disease. Cerebrovasc Dis. 2020 Jan;17:1-7.

2 Muraoka S, Araki Y, Kondo G, Kurimoto M, Shiba Y, Uda K, et al. Postoperative Cerebral Infarction Risk Factors and Postoperative Management of Pediatric Patients with Moyamoya Disease. World Neurosurg. 2018 May; 113:e190-9.
3 Tashiro R, Fujimura M, Kameyama M, Mugikura S, Endo H, Takeuchi Y, et al. Incidence and Risk Factors of the Watershed Shift Phenomenon after Superficial Temporal Artery-Middle Cerebral Artery Anastomosis for Adult Moyamoya Disease. Cerebrovasc Dis. 2019;47(3-4): 178-87.

4 Zhang J, Li S, Fujimura M, Lau TY, Wu X, Hu $\mathrm{M}$, et al. Hemodynamic analysis of the recipient parasylvian cortical arteries for predict- ing postoperative hyperperfusion during STA-MCA bypass in adult patients with moyamoya disease. J Neurosurg. 2019 Dec;27:18.

5 Bonasia S, Ciccio G, Smajda S, Weil AG, Chaalala C, Blanc R, et al. Angiographic Analysis of Natural Anastomoses between the Posterior and Anterior Cerebral Arteries in Moyamoya Disease and Syndrome. AJNR Am J Neuroradiol. 2019 Dec;40(12):206672. 